\title{
COVID-19: Real-time dissemination of scientific information to fight a public health emergency of international concern
}

\author{
Peipei Song', Takashi Karako ${ }^{2,3, *}$ \\ ${ }^{1}$ Institute for Global Health Policy Research, Bureau of International Health Cooperation, National Center for Global Health and Medicine, Tokyo, \\ Japan; \\ ${ }^{2}$ International Health Care Center, National Center for Global Health and Medicine, Tokyo, Japan; \\ ${ }^{3}$ Department of Surgery, the University of Tokyo Hospital, Tokyo, Japan.
}

SUMMARY Rapidly sharing scientific information is an effective way to reduce public panic about COVID-19, and doing so is the key to providing real-time guidance to epidemiologists working to contain the outbreak, clinicians managing patients, and modelers helping to understand future developments and the possible effectiveness of various interventions. This issue has rapidly reviewed and published articles describing COVID-19, including the drug treatment options for SARS-CoV-2, its clinical characteristics, and therapies involving a combination of Chinese and Western medicine, the efficacy of chloroquine phosphate in the treatment of COVID-19 associated pneumonia according to clinical studies, and reflections on the system of reserve medical supplies for public health emergencies. As an academic journal, we will continue to quickly and transparently share data with frontline healthcare workers who need to know the epidemiological and clinical features of COVID-19.

Keywords 2019-nCoV, SARS-CoV-2, COVID-19, sharing data

A novel coronavirus, formerly designated $2019-\mathrm{nCoV}$ and now taxonomically termed SARS-CoV-2, emerged in Wuhan, China at the end of 2019 and rapidly spread through many countries in Asia and elsewhere worldwide. This incident was labeled a public health emergency of international concern (PHEIC) on Jan. 30, 2020 (1). This is the 6th time WHO has declared a PHEIC since the International Health Regulations (IHR) came into effect in 2005.

The clinical condition caused by SARS-CoV-2 has been designated COVID-19 by the WHO. As of Feb. 23,2020 , WHO reported a total of 77,042 confirmed cases and 2,445 deaths in China and 1,769 confirmed cases and 17 deaths in 28 other countries (2). The rapid increase in infections and deaths caused anxiety, panic, stigma, mistrust, and rumor-mongering among public.

Rapidly sharing scientific information is an effective way to reduce public panic, and it is the key to providing real-time guidance to epidemiologists working to contain the outbreak, clinicians managing patients, and modelers helping to understand future developments and the possible effectiveness of various interventions.

This information includes routes of transmission and transmissibility, the natural history of infection in humans, the populations at risk, the successful clinical practices that are being used to manage patients, the laboratory information needed to diagnose patients, and the genetic sequence information used to assess viral stability.

Scientists from numerous countries have published and analyzed the genome of the pathogen responsible, SARS-CoV-2 (3-5). On Jan. 2020, a study that analyzed data on the first 425 confirmed cases in Wuhan, China provided evidence of human-to-human transmission among close contacts and suggested that measures to prevent or reduce transmission be implemented in populations at risk (6). Two studies published in Jan. 2020 described the epidemiological and clinical features of laboratory-confirmed COVID-19 in 41 and 99 patients, respectively, in Wuhan $(7,8)$. On Feb. 9, 2020, a study involving 1,099 laboratory-confirmed cases at 552 hospitals was published online to provide an up-to-date delineation of the epidemiological and clinical characteristics of COVID-19 throughout mainland China (9). The study's findings encourage a shift in focus to identifying and managing patients at an earlier stage, before disease progression. In addition to confirmed cases from China, several reports identified clusters of locally transmitted cases in other countries, adding to the understanding of this disease. 
To prompt real-time sharing of scientific information, many international academic journals have rapidly reviewed and published articles on COVID-19. Several coronavirus websites have also been created to assemble and disclose articles on this disease, such as the NEJM Coronavirus page, the Lancet COVID-19 Resource Centre, and the Cell Press Coronavirus Resource Hub. In order to overcome the language barrier, many articles have also been translated into Chinese to directly benefit frontline health professionals and policy makers in China as well as to reduce public panic; epidemiological information and scientific articles in Chinese have also been widely cited and rapidly reported in English. Broad dissemination in both Chinese and English will accomplish the goal of promptly communicating crucial findings to the international scientific community.

We still have a lot to learn about SARS-CoV-2 and the disease it causes, COVID-19. The real-time dissemination of scientific information is needed most during this period of uncertainty. Academic journals are responsible for facilitating the rapid dissemination of reliable information including transparent methods of identifying cases, sharing data, unfettered communication, and peer-reviewed research.

Since its inception in 2007, our journal - BioScience Trends - has highlighted the research on and dissemination of information regarding public health emergencies, leading us to publish articles on topics such as SARS, Ebola, MERS, avian influenza A H5N1, H1N1, and H7N9.

In this issue, we rapidly reviewed and published articles describing COVID-19, including the drug treatment options for SARS-CoV-2, its clinical characteristics, and therapies involving a combination of Chinese and Western medicine, the efficacy of chloroquine phosphate in the treatment of COVID-19 associated pneumonia according to clinical studies, and the reflections on the system of reserve medical supplies for public health emergencies.

As an academic journal, we will continue to quickly and transparently share data with frontline healthcare workers who need to know the epidemiological and clinical features of COVID-19.

\section{References}

1. World Health Organization Europe. 2019-nCoV outbreak is an emergency of international concern. httsp://www. euro.who.int/en/health-topics/health-emergencies/ international-health-regulations/news/news/2020/2/2019ncov-outbreak-is-an-emergency-of-international-concern (accessed February 22, 2020)

2. World Health Organization. Coronavirus disease 2019 (COVID-19) Situation Report - 33. https:// www.who.int/docs/default-source/coronaviruse/ situation-reports/20200222-sitrep-33-covid-19. $p d f ? s f v r s n=c 9585 c 8 f 2$ (accessed February 23, 2020)

3. $\mathrm{Wu} \mathrm{F}$, Zhao S, Yu B, et al. A new coronavirus associated with human respiratory disease in China. Nature. 2020; doi: 10.1038/s41586-020-2008-3.

4. Zhou P, Yang XL, Wang XG, et al. A pneumonia outbreak associated with a new coronavirus of probable bat origin. Nature. 2020; doi: 10.1038/s41586-020-2012-7.

5. Gorbalenya AE, Baker SC, Baric RS, et al. Severe acute respiratory syndrome-related coronavirus: the species and its viruses - A statement of the Coronavirus Study Group. bioRxiv preprint. Published online February 11, 2020; doi: https://doi.org/10.1101/2020.02.07.937862.

6. Li Q, Guan X, Wu P. Early transmission dynamics in Wuhan, China, of novel coronavirus-infected pneumonia. N Engl J Med. 2020; doi: 10.1056/NEJMoa2001316.

7. Huang C, Wang Y, Li X, et al. Clinical features of patients infected with 2019 novel coronavirus in Wuhan, China. Lancet. 2020; 395:497-506.

8. Chen N, Zhou M, Dong X, Qu J, Gong F, Han Y, Qiu Y, Wang J, Liu Y, Wei Y, Xia J, Yu T, Zhang X, Zhang L. Epidemiological and clinical characteristics of 99 cases of 2019 novel coronavirus pneumonia in Wuhan, China: A descriptive study. Lancet. 2020; 395:507-513.

9. Guan WJ, Ni ZY, Hu Y, et al. Clinical characteristics of 2019 novel coronavirus infection in China. medRxiv preprint. Published online February 9, 2020; doi: https:// doi.org/10.1101/2020.02.06.20020974.

Received February 23, 2020; Accepted February 24, 2020.

\section{*Address correspondence to:}

Takashi Karako, International Health Care Center, National Center for Global Health and Medicine, Tokyo, 1-21-1 Toyama Shinjuku-ku, Tokyo 162-8655, Japan.

E-mail: politang-tky@umin.ac.jp

Released online in J-STAGE as advance publication February 25, 2020. 